\title{
Promoting education within the SAPC: introducing the HoTs Group
}

\author{
Joe Rosenthal ${ }^{1}$, Bob McKinley ${ }^{2}$ and David Pearson ${ }^{3}$ \\ ${ }^{1}$ Department of Primary Care Population Health, University College London, London, UK \\ ${ }^{2}$ Arthritis Research UK Primary Care Centre, Keele University, Keele, UK \\ ${ }^{3}$ Academic Unit of Primary Care, University of Leeds, UK
}

The Society for Academic Primary Care (SAPC) exists to support, promote and develop the discipline of academic primary care. One of its stated key principles is the promotion of excellent teaching, research and critical reflection on primary care practice and policy.

As some readers may recall, the original precursor of the SAPC was the AUTGP (Association of University Teachers of General Practice), later rebranded AUDGP (Association of University Departments of General Practice). Over the course of this evolution from AUTGP to SAPC, there has been a sense among at least some General Practice educators that the focus of the Society has shifted away from education and training in favour of research and development. Although there is no question of the importance of the latter, the former remain core activities and major sources of funding for most academic primary care departments. Indeed, as primary care research has become increasingly specialised, it could be argued that it is primary care education that provides the common thread to bind our discipline together and protect academic departments from potential fragmentation into alternative clinical or methodological groupings.

The major contribution of primary care to the UK undergraduate medical curriculum in particular should not be underestimated. Most medical schools now deliver at least $15 \%$ of their teaching in the community. This contribution often goes beyond traditional General Practice (GP) placements, with SAPC members increasingly involved in areas such as curriculum development, quality assurance, assessment, admissions, welfare, as well as in senior educational leadership positions. The opportunities

Correspondence to: Dr Joe Rosenthal, Department of Primary Care \& Population Health, University College London Medical School, Royal Free Campus, Rowland Hill Street, London NW3 2PF, UK. Email: j.rosenthal@ucl.ac.uk for the SAPC to increase its educational role are also increasing in the postgraduate arena as deaneries restructure and focus increasingly on commissioning rather than providing education and training. The anticipated demise of Primary Care Trusts leaves a vacuum of leadership for continuing professional development among established primary care professionals including GPs, nurses and allied health professionals.

All good reasons to create new national Heads of Primary Care Teaching (HoTs) Group within the SAPC. First conceived in 2009, we aim to represent the interests of all primary care educators within medical schools around the United Kingdom, and to support the SAPC in achieving its aims in relation to primary care education and educational research. Membership of the HoTs Group currently consists of the heads of primary care teaching of all UK medical schools. The group meets twice a year, between which time our email forum provides a lively mode of exchange of information and ideas.

Future activity intends to build on this strong foundation. Our immediate priorities are to address concerns of members in relation to delivering highquality undergraduate medical education and building on the position of strength that our pivotal role within medical schools now affords us. In addition, we aim, over the next two years, to address our goals around scholarship and professional development, the encouragement of high-quality educational research, and the strengthening of our contribution to postgraduate medical education. Our initial focus is admittedly on undergraduate general practice education, but we do aim over time to broaden our remit and representation to support education and training within other primary care disciplines.

We should be very happy to hear from any SAPC members at any time and look forward to working together to promote the Society and the discipline of Academic Primary Care. 